\title{
Multiscattering inversion for low-model wavenumbers
}

\author{
Tariq Alkhalifah ${ }^{1}$ and Zedong $\mathrm{Wu}^{1}$
}

\begin{abstract}
A successful full-waveform inversion implementation updates the low-wavenumber model components first for a proper description of the wavefield propagation and slowly adds the high wavenumber potentially scattering parts of the model. The low-wavenumber components can be extracted from the transmission parts of the recorded wavefield emanating directly from the source or the transmission parts from the single- or double-scattered wavefield computed from a predicted scatter field acting as secondary sources. We use a combined inversion of data modeled from the source and those corresponding to single and double scattering to update the velocity model and the component of the velocity (perturbation) responsible for the single and double scattering. The combined inversion helps us access most of the potential model wavenumber information that may be embedded in the data. A scattering-angle filter is used to divide the gradient of the combined inversion, so initially the high-wavenumber (low-scattering-angle) components of the gradient are directed to the perturbation model and the low-wavenumber (highscattering-angle) components are directed to the velocity model. As our background velocity matures, the scatteringangle divide is slowly lowered to allow for more of the higher wavenumbers to contribute the velocity model. Synthetic examples including the Marmousi model are used to demonstrate the additional illumination and improved velocity inversion obtained when including multiscattered energy.
\end{abstract}

\section{INTRODUCTION}

Recorded seismic data are inherently dependent on the shortwavelength components of the earth model for the presence of most of the recorded events and dependent on the long-wavelength components for the geometric features of such events. In inverting for a velocity model, as the inversion process demands, we use the geometric features of the wavefield to extract the long-wavelength components first, which is in turn used to place the scattered events in their accurate position in what we typically refer to as imaging (Claerbout, 1985). Likewise, in full-waveform inversion (FWI), we use the extracted model from the geometric behavior or the transmission components to place the perturbations in their accurate locations in the velocity model in a frequency continuation process to accommodate inaccuracies in the propagator velocity (Bunks et al., 1995; Pratt et al., 1996; Virieux and Operto, 2009). The key element in the inversion strategy is the model wavenumber build up. Thus, FWI conventionally requires that every model wavenumber component should be placed (controlled almost uniformly by the propagator velocity) in the model in a location in which the resulting scattering is within a half-cycle of its true location in the data. For a certain, initially inaccurate, propagator velocity, this can be achieved for a low enough model wavenumber. These newly added wavenumbers tend to improve the propagator velocity so that higher frequencies may meet the half-cycle threshold (Bunks et al., 1995). From the data standpoint, the wavenumber continuation is commonly controlled by inverting first for low frequencies and large offset to depth ratios (Bunks et al., 1995; Pratt et al., 1996; Virieux and Operto, 2009), which can be achieved by damping later arrivals (Jang et al., 2009).

Recently, many authors have explored the wavenumber continuation theme from the model standpoint, instead of the data (Albertin et al., 2013; Almomin, 2013; Tang et al., 2013). Despite the value of data decimation and selection, the real objective of this process is far more apparent in the model domain and specifically at the gradient level (Sirgue and Pratt, 2004). Such developments resulted into a more direct control of the model wavenumber update, given by a scattering-angle filter (Alkhalifah, 2015b). Scattering-angle filtering provided a directional independent filter of the gradient valid for FWI and migration velocity analysis (MVA) gradients. Unlike a classic smoothing operator, the scattering-angle filter is governed by a physical quantity of the scattering process providing low wavenumbers along even complex raypaths, resulting from a complex

\footnotetext{
Manuscript received by the Editor 21 November 2015; revised manuscript received 3 July 2016; published online 21 September 2016.

${ }^{1}$ King Abdullah University of Science and Technology, Physical Sciences and Engineering, Thuwal, Saudi Arabia. E-mail: tariq.alkhalifah@kaust.edu.sa; zedong.wu@kaust.edu.sa.

(C) 2016 Society of Exploration Geophysicists. All rights reserved.
} 
background velocity. This is provided by its velocity-dependent smoothing operator, more easily implemented in the wavenumber domain using an extended normalized time axis. It is also more adaptable to multiparameter inversion in which we can apply an independent scattering-angle filter to each of the parameters (Alkhalifah, 2015a).

However, a proper continuation of the wavenumbers also requires their presence in, or their proper extraction from, the data. To do so, we need to optimize the conventionally modeled data, as well as those that rely on the image (Almomin and Biondi, 2012; Berkhout, 2012; Fleury and Perrone, 2012; Ma et al., 2012; Xu et al., 2012; Albertin et al., 2013; Wang et al., 2013). Low-wavenumber information extracted directly from the data residual has typically mild depth penetration limited by the penetration of the diving waves. However, we can also extract low wavenumbers from the data by imaging the reflections. We can either use extended images for classic MVA (Symes and Kern, 1992) or apply a demigration to implement what is referred to as reflection waveform inversion (RWI) (Chavent and Plessix, 1999; Clement et al., 2001; Xu et al., 2012).

In this paper, we model the direct source, as well as the single and double (including multiple) scattered, wavefields to contribute to the predicted data as we compare them with the observed data using a least-squares objective functional (as an example). Although the modeled source wavefield depends only on the velocity model, the single- and double-scattered wavefields depend on the velocity and perturbation models. Using a combined objective functional, we invert for velocity and perturbation models. The perturbation model produces reflections and double scattering only when they are not predicted by the modeled wavefield from the source using the velocity model. A scattering-angle filter is used to control the wavenumber distribution between the background velocity and perturbation models. In this case, the gradient is split into a part more appropriate initially for the velocity model (low wavenumbers) and a part needed for the perturbation model to generate the scattered wavefield.

\section{FWI AND RWI}

FWI usually starts with a reasonably smooth velocity model incapable of producing the needed scattering (reflections) we observe in the recorded data, so we tend to attenuate such reflections in the data in the initial stages, sometimes in all stages, of FWI (or what is referred to as waveform tomography) to avoid the considerable nonlinearity brought about by the data mismatch (Pratt, 1999). The RWI generates the first-order scattering, even with an initial smooth velocity model, using a migration/demigration process (Zhou et al., 2015). However, the comparison in this case is dominated by the geometric aspects of the wavefields, not the velocity's capability of producing such reflections, and thus, it provides mainly smooth wavepath updates. To understand the role of RWI and FWI, let us first look into their gradients.

Starting from the definition of the classic FWI objective functional (Tarantola, 1984)

$$
E_{\mathrm{FWI}}(m)=\sum_{i}\left|d_{o_{i}}-d_{s_{i}}(m)\right|^{2}
$$

we define $i$ as the source index, $d_{o}$ as the observed data, $d_{s}=u_{s}\left(\mathbf{x}=\mathbf{x}_{\mathbf{r}_{\mathbf{j}}}, t\right)$ as the modeled synthetic data, $m(\mathbf{x})$ as the velocity model described in space using the vector $\mathbf{x}=\{x, y, z\}$, along the Cartesian coordinates, and $\mathbf{x}_{\mathbf{r}_{\mathbf{j}}}$ are the receiver locations for receiver indexed by $j$ with $t$ as time. From this point on, and for simplicity, but without loss of generality, we represent wavefields and data in the frequency domain. In this case, the modeled wavefield $u_{s}$ satisfies the following wave equation:

$$
L(m) u_{s_{i}}=f(\omega) \delta\left(\mathbf{x}-\mathbf{x}_{s_{i}}\right)
$$

for a particular source location $\mathbf{x}_{s_{i}}$, and $f$ is the source function given in the frequency domain. The modeling operator is given by

$$
L(m)=L^{t}(m)=\nabla^{2}+\omega^{2} m(\mathbf{x}),
$$

which in this case is self adjoint, with the adjoint operator $L^{t}, \omega$ is the angular frequency, $\nabla$ is the gradient, and $m=\left(1 / v^{2}\right)$. The gradient of the objective function (Tarantola, 1984) is given by

$$
R_{\mathrm{FWI}}(\mathbf{x})=\nabla_{m} E_{\mathrm{FWI}}=\Re\left\{-\frac{\partial L}{\partial m}\left(=\omega^{2}\right) \sum_{i} u_{s_{i}}(\mathbf{x}) u_{r 1_{i}}(\mathbf{x})\right\},
$$

where $\Re$ stands for the real part, and

$$
L(m) u_{r 1_{i}}=\sum_{j} \delta\left(\mathbf{x}-\mathbf{x}_{r_{j}}\right)\left(d_{o_{i j}}-d_{s_{i j}}\right)^{*}
$$

where $\mathbf{x}_{r_{j}}$ are the locations of the receivers for a particular receiver $j$.

However, we can produce data directly from the perturbed part of the velocity model $\delta m$ (or the image) and use the following objective function (Clement et al., 2001; Xu et al., 2012):

$$
E_{\mathrm{RWI}}(m, \delta m)=\sum_{i}\left|d_{o_{i}}-d_{m_{i}}(m, \delta m)\right|^{2}
$$

where $d_{m}$ is the modeled data from the perturbation $\delta m(\mathbf{x})$. From the inverse Born approximation, the perturbation is given by

$$
\delta m(\mathbf{x})=\nabla_{\delta m} E_{\mathrm{RWI}}=\Re\left\{-\omega^{2} \sum_{i} u_{s_{i}}(\mathbf{x}) u_{a_{i}}(\mathbf{x})\right\},
$$

where the receiver wavefield $u_{a_{i}}$ satisfies

$$
L^{t}(m) u_{a_{i}}=\sum_{j} \delta\left(\mathbf{x}-\mathbf{x}_{r_{j}}\right) d_{o_{i j}}^{*}
$$

and thus, equation 7 provides the image. As a result, we can use the predicted perturbations along with the Born scattering approximation to solve for the scattered wavefield $\delta u_{s_{i}}$ using the following equation (Zhang and Duan, 2012):

$$
L(m) \delta u_{s_{i}}=-\omega^{2} \delta m(\mathbf{x}) u_{s_{i}}(\mathbf{x})
$$

and $d_{m_{i}}=\delta u_{s_{i}}\left(\mathbf{x}_{r_{i}}\right)$.

The gradient corresponding to this objective function with respect to the model is given by (Wang et al., 2013) 


$$
\begin{aligned}
R_{\mathrm{RWI}}(\mathbf{x}) & =\nabla_{m} E_{\mathrm{RWI}} \\
& =\Re\left\{-\omega^{2} \sum_{i}\left(u_{s_{i}}(\mathbf{x}) \delta u_{r_{i}}(\mathbf{x})+u_{r 2_{i}}(\mathbf{x}) \delta u_{s_{i}}(\mathbf{x})\right)\right\}
\end{aligned}
$$

where

$$
\begin{gathered}
L^{t}(m) u_{r 2_{i}}=\sum_{j} \delta\left(\mathbf{x}-\mathbf{x}_{r_{j}}\right)\left(d_{o_{i j}}-d_{m_{i j}}\right)^{*}, \\
L^{t}(m) \delta u_{r_{i}}=-\omega^{2} \delta m(\mathbf{x}) u_{r_{i}}(\mathbf{x}) .
\end{gathered}
$$

The two terms in equation 10 correspond to the source- and receiver-side wavepath gradients. A detailed analysis of this gradient is given by Alkhalifah and Wu (2016).

\section{MULTISCATTERING INVERSION}

Alkhalifah and Wu (2016) combine FWI and RWI into a single objective function that naturally adhered to both objectives, FWI and RWI. Accordingly, we add the, reasonably important for salt flanks, double-scattering (or multiscattering, with inversion MWI) component to suggest the following objective function along the lines they suggested:

$$
\begin{aligned}
& E_{\mathrm{MWI}}(m, \delta m) \\
& \quad=\frac{1}{2} \sum_{i}\left|d_{o_{i}}-d_{s_{i}}(m)-d_{m 1_{i}}(m, \delta m)-d_{m 2_{i}}(m, \delta m)\right|^{2},
\end{aligned}
$$

where $d_{m 2_{i}}(m, \delta m)$ is the double-scattering data extracted from the double-scattered wavefield $\delta^{2} u_{s}\left(\mathbf{x}_{\mathbf{r}}\right)$ through $d_{m 2_{i}}=\delta^{2} u_{s_{i}}\left(\mathbf{x}_{\mathbf{r}}\right)$, with $d_{m 1_{i}}(m, \delta m)=d_{m}$ from the previous section. The double-scattered wavefield satisfies (Zhang and Duan, 2012)

$$
L(m) \delta^{2} u_{s_{i}}=-\omega^{2} \delta m(\mathbf{x}) \delta u_{s_{i}}(\mathbf{x}),
$$

which is a reformulation of the double-scattering Born term. Similar to its single-scattering counterpart (Alkhalifah and $\mathrm{Wu}, 2016$ ), any reflections or double-scattered multiples not predicted by the model $m$, will be generated in $d_{m 1}$ and $d_{m 2}$, respectively.

To obtain the corresponding gradients, we use the extended Lagrangian formulation (Plessix, 2006)

$$
E\left(m, \delta m, u_{s}, \delta u_{s}, \lambda_{1}, \lambda_{2}, \lambda_{3}\right)=E_{\mathrm{MWI}}\left(u_{s}, \delta u_{s}\right)-A_{1}-A_{2}-A_{3},
$$

where

$$
\begin{gathered}
A_{1}\left(m, u_{s}, \lambda_{3}\right)=\left\langle\lambda_{3}, L u_{s}-s\right\rangle, \\
A_{2}\left(m, \delta m, u_{s}, \delta u_{s} \lambda_{2}\right)=\left\langle\lambda_{2}, L \delta u_{s}+\omega^{2} \delta m u_{s}\right\rangle, \\
A_{3}\left(m, \delta m, u_{s}, \delta u_{s}, \delta^{2} u_{s}, \lambda_{1}\right)=\left\langle\lambda_{1}, L \delta^{2} u_{s}+\omega^{2} \delta m \delta u_{s}\right\rangle,
\end{gathered}
$$

and $\langle$.$\rangle stands for the dot product over space (because our wave-$ fields are in the frequency domain). The reason for the reverse order in the index of $\lambda_{i}$ will be apparent in deriving the gradient. Our objective is given by the stationary of the Lagrangian with respect to the state and adjoint-state variables, and thus, we set the gradient of $E$ with respect to all these variables to zero. The state equations are obvious and given in equations 2,9 , and 14 , and thus, we focus on the adjoint-state formulas obtained by setting the following to equal zero:

$$
\frac{\partial E}{\partial u_{s}}=-L^{t} \lambda_{3}-\omega^{2} \delta m \lambda_{2}+\sum_{j} \Delta d\left(\mathbf{x}_{\mathbf{r}_{\mathbf{j}}}\right) \delta\left(\mathbf{x}-\mathbf{x}_{\mathbf{r}_{\mathbf{j}}}\right),
$$

$$
\frac{\partial E}{\partial \delta u_{s}}=-L^{t} \lambda_{2}-\omega^{2} \delta m \lambda_{1}+\sum_{j} \Delta d\left(\mathbf{x}_{\mathbf{r}_{\mathbf{j}}}\right) \delta\left(\mathbf{x}-\mathbf{x}_{\mathbf{r}_{\mathbf{j}}}\right),
$$

$$
\frac{\partial E}{\partial \delta^{2} u_{s}}=-L^{t} \lambda_{1}+\sum_{j} \Delta d\left(\mathbf{x}_{\mathbf{r}_{\mathbf{j}}}\right) \delta\left(\mathbf{x}-\mathbf{x}_{\mathbf{r}_{\mathbf{j}}}\right)
$$

where $\Delta d=d_{o_{i}}-d_{s_{i}}(m)-d_{m 1_{i}}(m, \delta m)-d_{m 2_{i}}(m, \delta m)$. Reorganizing these equations in the order, they would be evaluated yields

$$
L^{T} \lambda_{1}=\sum_{j} \Delta d\left(\mathbf{x}_{\mathbf{r}_{\mathbf{j}}}\right) \delta\left(\mathbf{x}-\mathbf{x}_{\mathbf{r}_{\mathbf{j}}}\right)
$$

$L^{T} \lambda_{2}=-\omega^{2} \delta m \lambda_{1}+\sum_{j} \Delta d\left(\mathbf{x}_{\mathbf{r}_{\mathbf{j}}}\right) \delta\left(\mathbf{x}-\mathbf{x}_{\mathbf{r}_{\mathbf{j}}}\right)=-\omega^{2} \delta m \lambda_{1}+L^{T} \lambda_{1}$,

$L^{T} \lambda_{3}=-\omega^{2} \delta m \lambda_{2}+\sum_{j} \Delta d\left(\mathbf{x}_{\mathbf{r}_{\mathbf{j}}}\right) \delta\left(\mathbf{x}-\mathbf{x}_{\mathbf{r}_{\mathbf{j}}}\right)=-\omega^{2} \delta m \lambda_{2}+L^{T} \lambda_{1}$.

These adjoint wavefields $\left\{\lambda_{1}, \lambda_{2}, \lambda_{3}\right\}$ correspond to the residual wavefield, and its combination with its first- and second-order scattering, respectively. Thus, we can define $\lambda_{2}^{\prime}=\lambda_{2}-\lambda_{1}$ and $\lambda_{3}^{\prime}=$ $\lambda_{3}-\lambda_{1}-\lambda_{2}^{\prime}$, and as a result

$$
L^{T} \lambda_{1}=\sum_{j} \Delta d\left(\mathbf{x}_{\mathbf{r}_{\mathbf{j}}}\right) \delta\left(\mathbf{x}-\mathbf{x}_{\mathbf{r}_{\mathbf{j}}}\right)
$$

$$
L^{T} \lambda_{2}^{\prime}=-\omega^{2} \delta m \lambda_{1}
$$

$$
L^{T} \lambda_{3}^{\prime}=-\omega^{2} \delta m \lambda_{2}^{\prime}
$$

which represents the adjoint wavefields corresponding to a single source type. Therefore, now the adjoint wavefields $\left\{\lambda_{1}, \lambda_{2}^{\prime}, \lambda_{3}^{\prime}\right\}$ 
correspond to the residual wavefield, its first-order scattering, and its second-order scattering, respectively.

Finally, taking the derivative of $E$ with respect to the model parameters, where the state and adjoint-state variables are considered fixed, we obtain

$$
\begin{gathered}
\frac{\partial E}{\partial m}=\frac{\partial E_{\mathrm{MWI}}}{\partial m}=-\omega^{2}\left(u_{s} \lambda_{3}+\delta u_{s} \lambda_{2}+\delta^{2} u_{s} \lambda_{1}\right), \\
\frac{\partial E}{\partial \delta m}=\frac{\partial E_{\mathrm{MWI}}}{\partial \delta m}=-\omega^{2}\left(u_{s} \lambda_{2}-\delta u_{s} \lambda_{1}\right) .
\end{gathered}
$$

Taking the real part of the gradients with respect to the two model parameters and rearranging slightly

$$
\begin{aligned}
\nabla_{m} E_{\mathrm{MWI}}= & \Re\left\{-\omega^{2}\left(u_{s} \lambda_{3}+\delta u_{s} \lambda_{2}+\delta^{2} u_{s} \lambda_{1}\right)\right\}, \\
= & \Re\left\{-\omega^{2}\left(u_{s} \lambda_{1}+u_{s} \lambda_{2}^{\prime}+\delta u_{s} \lambda_{1}+u_{s} \lambda_{3}^{\prime}\right.\right. \\
& \left.\left.+\delta u_{s} \lambda_{2}^{\prime}+\delta^{2} u_{s} \lambda_{1}\right)\right\}, \\
\nabla_{\delta m} E_{\mathrm{MWI}}= & \Re\left\{-\omega^{2}\left(u_{s} \lambda_{2}+\delta u_{s} \lambda_{1}\right)\right\}, \\
= & \Re\left\{-\omega^{2}\left(u_{s} \lambda_{1}+u_{s} \lambda_{2}^{\prime}+\delta u_{s} \lambda_{1}\right)\right\},
\end{aligned}
$$

where $\Re$ stands for the real part. If we look at the gradient with respect to the model carefully, we realize it includes the gradients corresponding to FWI and RWI, and we can write it in this form

$$
\nabla_{m} E_{\mathrm{MWI}}=R_{\mathrm{FWI}}+R_{\mathrm{RWI}}-\Re\left\{\omega^{2}\left(u_{s} \lambda_{3}^{\prime}+\delta u_{s} \lambda_{2}^{\prime}+\delta^{2} u_{s} \lambda_{1}\right)\right\},
$$

with the last three terms corresponding to the multiscattering terms. In fact, the gradient of the perturbation part is given by the FWI and RWI gradients. The image gradient includes the classic leastsquares image with the contribution of the double-scattering imaging term.

\section{INVERSION OPTIONS}

From the gradients of $E_{\mathrm{MWI}}$ with respect to the model and perturbation, shown in equations 30 and 31 , we have multiple options in building our velocity model. A simultaneous inversion of $m$ and $\delta m$, as independent parameters, is one option (Wu and Alkhalifah, 2015). The approach given in equation 13 automatically handles multiscattering in the image. Interestingly, the image after optimization is also an optimal gradient to the inversion because it includes the double-scattering component of the full Hessian.

Another option is to extract the update from the perturbation model. After some iterations of optimizing only $\delta m$ for a given background $m$ using equation 31, we fix it to compute the gradient $m$ using equation 30 , in a nested loop-type implementation. This implementation has roots in the truncated Newton approach (Métivier et al., 2014), but our fitting here is applied directly to the data instead of the gradient. The cost is equivalent to solving for the update $\delta \mathbf{m}$ using

$$
\mathbf{H} \delta \mathbf{m}=-\nabla \mathbf{E},
$$

where $\mathbf{H}$ is the Hessian, with its action on $\delta \mathbf{m}$ computed using the double-scattering modeling described above. A conjugate gradient approach to solve equation 33 , is possible, and referred to as the truncated Newton approach (Métivier et al., 2014). Alternatively, the above may be formulated and is more complete by fitting the double scattering to the data directly, as we will see later.

\section{FILTERING THE GRADIENT}

Filtering the gradient using extended images does not only provide us with an opportunity to control the wavenumbers admitted to the velocity model, independent of frequency, but also provides the opportunity to clean up our gradients for the model and perturbation. In multiscattering, the large scattering angles may not correspond to large offsets any more, depending on the scattering path, but still it will provide the smoother wavepath elements we seek.

Thus, to improve the action of the gradient and specifically to allow it to focus on the appropriate long-wavelength components, we use a slightly modified time lag (velocity scaled $\zeta$; Khalil et al., 2013) to our gradients for FWI (Alkhalifah, 2015b)

$$
R(\mathbf{x}, \zeta)=R(\mathbf{x}) e^{-4 i \omega \frac{\zeta}{v(\mathbf{x})}}
$$

where $R$ stands for any one of the gradients discussed above. Here, $v$ is the velocity, $(1 / \sqrt{m})$, and $\zeta=(\tau / 2) v(\mathbf{x})$, where $\tau$ is the classic time lag, which explains the relation between time lags and this new distance lag. An inherent feature of this modified time-lag (distance units) representation is that the relationship between the scattering angle and the wavenumber of the gradient is free of a velocity (space) dependency. In fact, the scattering angle $\theta$ is then given by

$$
\cos ^{2} \frac{\theta}{2}=\frac{\left|\mathbf{k}_{\mathbf{m}}\right|^{2}}{k_{\zeta}^{2}}
$$

where $\mathbf{k}_{\mathbf{m}}$ is the model-wavenumber vector and $k_{\zeta}$ is the wavenumber (Fourier transform) corresponding to $\zeta$. The scattering-angle filter corresponding to a particular angle is linear, and thus, we can denote the operation by the symbol $\mathcal{L}_{s}$, in which

$$
\mathcal{L}_{s}\left(R_{1}+R_{2}\right)=\mathcal{L}_{s} R_{1}+\mathcal{L}_{s} R_{2}
$$

Because the action of a Hessian $\mathbf{H}$ on a desired update, $\delta \mathbf{m}$ is given by the gradient $\mathbf{R}$; specifically

$$
\mathcal{L}_{s} \mathbf{H} \delta \mathbf{m}=\mathcal{L}_{s} \mathbf{R}
$$

then the application of the scattering-angle filter in formula 37 can result in an update $\delta \mathbf{m}$ corresponding to the filtered gradient. The action of the Hessian on the desired update is given by Métivier et al. (2014)

$$
\mathbf{H} \delta \mathbf{m}=\Re\left\{-\frac{\partial L}{\partial m}\left(\delta u \lambda_{1}+u \lambda_{2}^{\prime}+u \lambda_{4}\right)\right\},
$$

where 


$$
L^{t} \lambda_{4}=\sum_{j} d_{m}\left(\mathbf{x}_{\mathbf{r}_{\mathbf{j}}}\right) \delta\left(\mathbf{x}-\mathbf{x}_{\mathbf{r}_{\mathbf{j}}}\right) .
$$

Hence, the application of the filter is distributive to all the components in equation 38 .

\section{COMBINING THE PIECES}

In the following, we describe an approach that uses the FWI/ RWI/MWI combination and the power of the scattering-angle filter. The approach is based on manipulating the gradient using a scattering-angle filter.

The process starts with an initial velocity model that is used to generate synthetic seismograms using, as much as we can, the conditions and experiment variables used in the field. We will, however, restrict the description here to the acoustic constant density case, for simplicity. The residual between the modeled and field data provides the source function for the adjoint-state field that we correlate with the modeled wavefield, usually at all model points. Using equation 34 , we extend the gradient along the normalized timelag dimension, which will allow us to access the scattering-angle information, as shown in equation 35 . We can also obtain the extended image with more accurate amplitude and better resolution using a least-squares inversion.

We then divide the gradient into two parts, one corresponding to low-scattering angles $R_{l}$, and the other corresponding to high-scattering angles $R_{h}$, as follows:

$$
R_{1}(\mathbf{x}, \zeta)=R_{l_{1}}(\mathbf{x}, \zeta)+R_{h_{1}}(\mathbf{x}, \zeta),
$$

where the subscript 1 stands for the single-scattering level. Thus, the gradients are given by $\hat{R}_{l_{1}}(\mathbf{x})=R_{l_{1}}(\mathbf{x}, 0)$ and $\hat{R}_{h_{1}}(\mathbf{x})=R_{h_{1}}(\mathbf{x}, 0)$. If the cutoff angle is high (near $180^{\circ}$ ), $\hat{R}_{h_{1}}$ will be dominated by only low-wavenumber information that can be added to the background velocity model. The residual $\hat{R}_{l_{1}}$ is then used in the RWI as the initial $\delta m$. This perturbation model should be free of low-frequency artifacts and possibly other noise (Khalil et al., 2013). This will result in a cleaner scattered field $\delta u_{s}$ and a cleaner RWI gradient $R_{2}(\mathbf{x})$. However, $R_{2}(\mathbf{x})$ may contain high-wavenumber components from multiscattering information in our data. It may also include additional high-wavenumber information if the adjoint-state method was used to develop the gradient instead of least-squares inversion. In this case, we apply another scattering-angle filter, but now, to the RWI gradient, using probably the same cutoff angle used in the first filter. We can then add the two smooth gradients as well as the sharp ones to update our velocity and perturbation models:

$$
\begin{aligned}
& m(\mathbf{x})=R_{h_{1}}(\mathbf{x}, 0)+R_{h_{2}}(\mathbf{x}, 0), \\
& \delta m(\mathbf{x})=R_{l_{1}}(\mathbf{x}, 0)+R_{l_{2}}(\mathbf{x}, 0) .
\end{aligned}
$$

We can also use $\delta m(\mathbf{x})$ to obtain a smooth gradient corresponding to wavepaths associated with multiscattering energy, by applying multiscattering modeling because we use $\delta u_{s}$ as the source wavefield in equation 14, and $\delta m(\mathbf{x})$ as the image as follows:

$$
L(m) \delta^{2} u_{s_{i}}=-\omega^{2} \delta m(\mathbf{x}) \delta u_{s_{i}}(\mathbf{x}) .
$$

The contribution from this step is expected to be small, an inherent feature of doubly scattered energy, unless we have salt flanks.

At later iterations, we slowly lower the scattering angle cutoff, which will allow more scattering energy in the velocity model through $R_{h_{1}}(\mathbf{x}, \zeta)$, and more double-scattering energy through $R_{h_{2}}(\mathbf{x}, \zeta)$, hopefully when the velocity model after the smooth updates (correct background) is capable of mapping them accurately. We summarize the steps involved in the proposed approach in the following:

1) Establish an initial background velocity $m$.

2) Compute $d_{s}$ by solving the wave equation.

3) We optimize $\delta m$ by fitting $d_{m}$ to $d_{o}-d_{s}$ as in equation 13 taking into consideration multiscattering, as $d_{m}=d_{m 1}+d_{m 2}+\ldots$, and allow mainly low-scattering angles (high-wavenember components) to contribute to $\delta m$.

4) Update $m$ using the smooth (high-scattering angle) components of the Born and multiscattering-based gradient of equation 30.

5) Repeat steps 2 and 4 until convergence.

Based on our objective function, an optimal convergence is achieved, if $\delta m$ is given by zero, in which all reflections are predicted by $m$. However, in real cases, $\delta m$ will hold the residuals that we were not able to fit to the data, due to many reasons such as using approximate physics.

\section{A SIMPLE GRADIENT TEST}

In this example, we demonstrate how we can split the gradient to a velocity and perturbation model components using a scatteringangle filter. We consider the velocity model as shown in Figure 1a. We acquired data from 40 shots on the surface with shot interval of $140 \mathrm{~m}$. The receivers are distributed along the surface at $20 \mathrm{~m}$ interval. Our background velocity model is constant throughout given by $2000 \mathrm{~m} / \mathrm{s}$. A conventional single scattering least-squares inversion (like imaging) of $\delta m$ is not capable of inverting for the vertical component of the perturbation as shown in Figure 1b. However, our perturbation model given by the double-scattering component (equation 31) as well managed to image the vertical piece of the interface (Figure 1c) - a feature most useful for salt flanks. In both cases, we apply a scattering-angle filter that attenuates large-scattering angles, which explains the clean nature of the images.

If we impose on the velocity gradient (equation 30 ) a high-pass scattering-angle filter, we obtain smooth updates corresponding to the direct arrival (first term of the equation), corresponding to single scattering (second and third terms), and corresponding to double scattering (the last three terms). As a result, Figure 2 shows a smooth update for a single source at $14 \mathrm{~km}$ location laterally and a receiver at $22 \mathrm{~km}$. In addition to the rabbit component, we are accustomed to with RWI (or any MVA approach based on single scattering), we obtain a lower amplitude component reflecting the double-scattering wavepath from the vertical reflector. This component adds valuable additional illumination to the background update. The value of this additional component will be realized later in inverting for the Marmousi model. Because a high-pass scattering angle cutoff near $180^{\circ}$ is applied, the gradient is considerably smooth. Thus, using scattering-angle filtering, we can split our computed gradients to a background model and a perturbation part. 


\section{THE ROLE OF DOUBLE SCATTERING}

Is double scattering enough? or should we use triple scattering? In addition to the obvious role that double scattering may play in
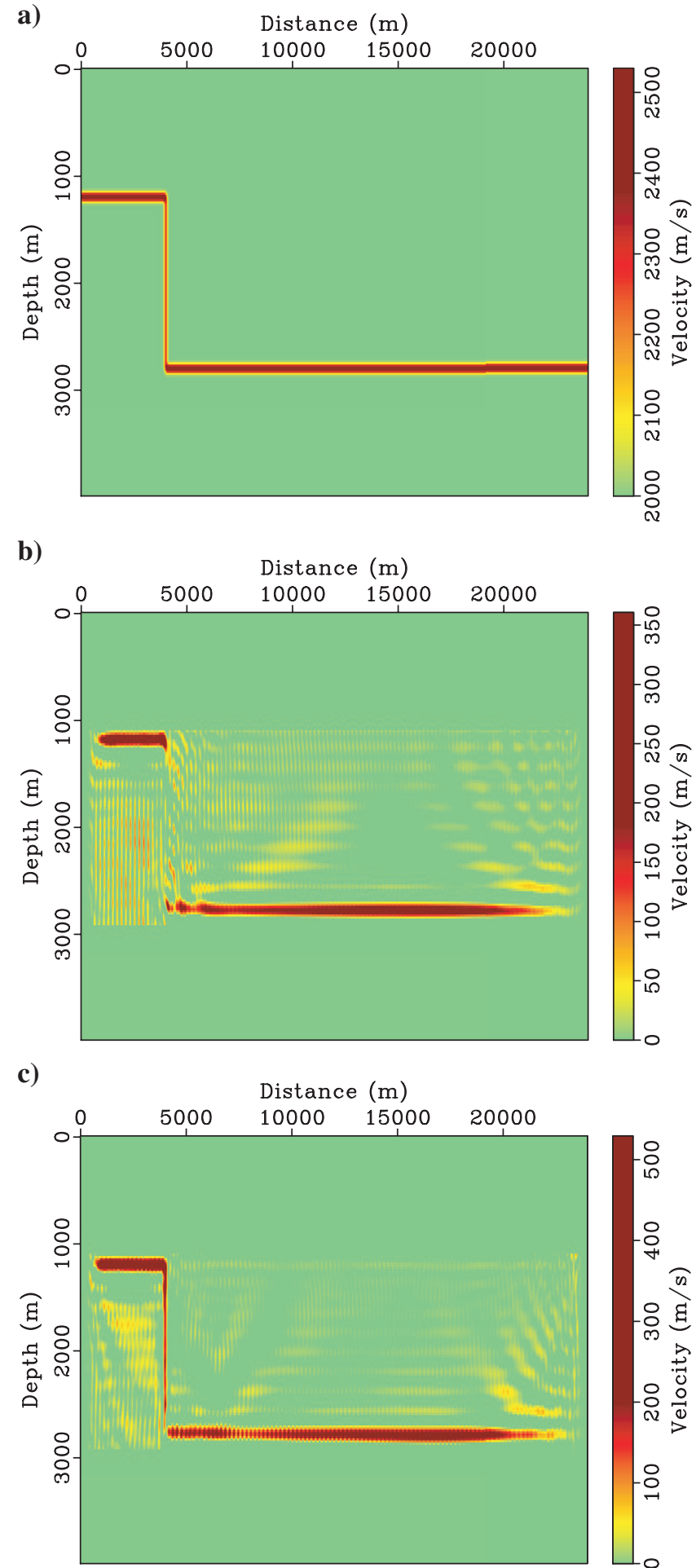

Figure 1. (a) The actual velocity model. (b) The perturbation model for single scattering obtained using the first term of equation 31. (c) The perturbation model corresponding to using all terms in equation 31. A low-pass scattering-angle filter with an angle $75^{\circ}$ is applied. inverting salt flanks, we can find models in which triple scattering might provide useful information, but is it worth the additional cost? We test the role that multiscattering plays in improving the convergence of FWI. We focus mainly on $\delta m$ in a purely FWI procedure. Because the scattering series requires small perturbations for convergence, we consider the model shown in Figure 3a. The background model $m_{0}$ is given by $1500 \mathrm{~m} / \mathrm{s}$. Although the perturbation is mild in magnitude $(100 \mathrm{~m} / \mathrm{s})$, it has a wide support (more than three times the wavelength in the extrapolated wavefield, which corresponds to data with frequency of $5 \mathrm{~Hz}$ ). We use a Helmholtz solver to compute the state and adjoint-state variables and invert for $\delta m$. The sources and receivers cover the top surface of the model.

In the first set of tests, we update only $\delta m$ to produce data that will ultimately fit the data corresponding to the exact model. Thus, $m_{0}$ will remain constant. In this case, the modeling operator remains the same for all the wavefields involved, and as such, the cost of the inversion is low because effectively only one matrix decomposition is needed in the Helmholtz solver. In Figure 3b, we show the result of adding a $\delta m$ to the background that is computed using 100 iterations of LBFGS updates to fit $d_{m 1}$ to $d_{o}$. This $\delta m$ is effectively the gradient we conventionally use in FWI with an approximate GaussNewton preconditioner. As expected, we mainly captured the top of the perturbation area, similar to what we obtain from imaging. If we decide to use also $d_{m 2}$ in the fitting process, $\delta m$ (added to $m_{0}$ ) looks much better with more infill in the center (Figure 3c). This $\delta m$ is similar to what we get from a full Newton preconditioner, but with an additional proper scaling for the second-order scattering given by usually the components of the third-order term of the Taylor's series expansion of the classic FWI objective function. In other words, we managed to include the proper scaling for double scattering. Again because the Helmholtz operator does not change, we can fit higher order scattering at little additional cost, and Figure 3d-3f shows the $m_{0}+\delta m$ for $\delta m$ corresponding to third-, fifth-, and twelfth-order scatterings, respectively, all evaluated using 100 iterations. The resulting $m$ looks similar to that from double scattering (Figure $3 \mathrm{c}$ ).

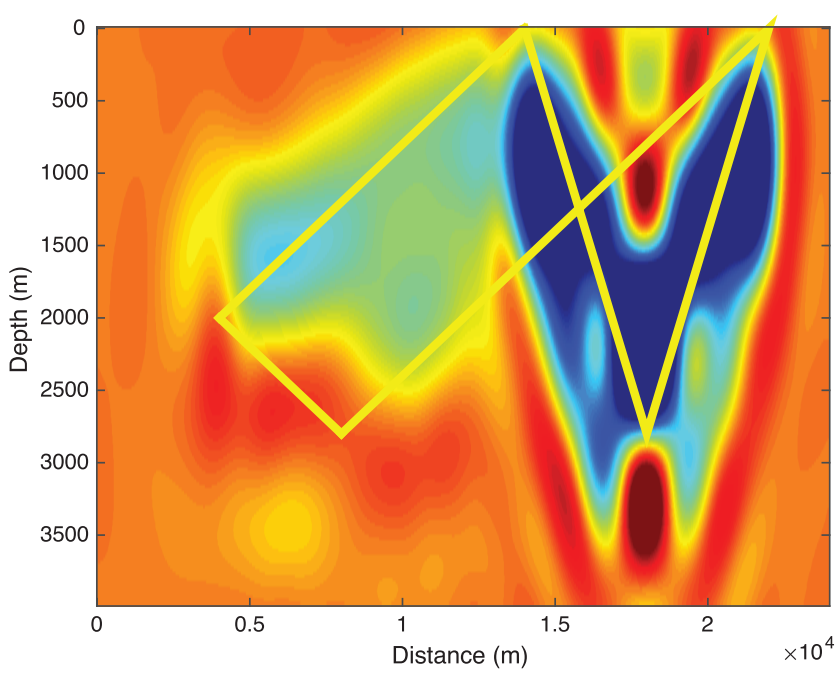

Figure 2. The gradient corresponding to the background for the double scattering (equation 30) for a single source and receiver. It shows a low-amplitude additional branch on the left beyond the familiar rabbit ear. The thick yellow lines show the corresponding raypaths. 
Therefore, the big improvement is from single to double scattering for this model.

If we decide to add $\delta m$ computed using single, double, and triple scattering to $m_{0}$, in an iterative fashion, the process becomes more like FWI. Because the Helmholtz operator needs to be computed every time we update $m_{0}$, we apply only 20 iterations of FWI. Figure $4 \mathrm{a}$ shows the inverted model corresponding to single scattering based $\delta m$ after the 20 iterations. Despite, the apparent, less than impressive, gradient of the first iteration (Figure $4 \mathrm{~b}$ ), the inversion managed to admit a reasonable model. This model is not that different from what we get from using the double scattering based $\delta m$ (Figure $4 \mathrm{~b})$. The same conclusion can be stated for the triple scattering based $\delta m$ (Figure $4 \mathrm{c}$ ) or even fifth-order scattering (Figure $4 \mathrm{~d}$ ). Thus, despite the improvement in $\delta m$ from double scattering, in the iterative update of $m_{0}$, the single-scattering update managed to converge to a reasonable result. This happens because the update of $m_{0}$ starts to predict the multiscattering in the background model, and thus, the multiscattering phenomena are taken care by $m_{0}$. Multiscattering consideration in the gradient helps FWI, but not as much as we hoped. It might have helped in converging faster (at least we see that in the first iteration given in Figure 3c-3e), but our focus here is information extraction not efficiency.
Thus, the real value of multiscattering in our implementation is its additional wavepath updates to $m_{0}$ along double-scattered paths, such as what we saw in Figure 2. The additional low-wavenumber illumination can help to provide low-wavenumbers updates in areas otherwise not covered by single scattering. In addition, wavepath updates between nearby scatterers provide reasonably high-resolution wavepath updates needed to fill in the middle-wavenumber components. These features will be demonstrated next on the Marmousi example.

\section{THE MARMOUSI MODEL}

We test the use of double scattering in inversion on the versatile Marmousi model (Figure 5a). We filter out of the generated observed data (corresponding to the exact model) frequencies less than $5 \mathrm{~Hz}$ to make the problem more challenging (a shot gather is shown in Figure 5b). The velocity $m_{0}$ and perturbation $\delta m$ models are sampled at $32 \times 32 \mathrm{~m}$. We consider shots every $256 \mathrm{~m}$ and receivers at the location of all the grid points on the surface. The initial linearly increasing velocity model is shown in Figure $6 \mathrm{a}$, and the initial $\delta m=0$, and as a result the residual data (Figure 6b) contain a lot of the reflection events not predicted by the initial model. We use a)

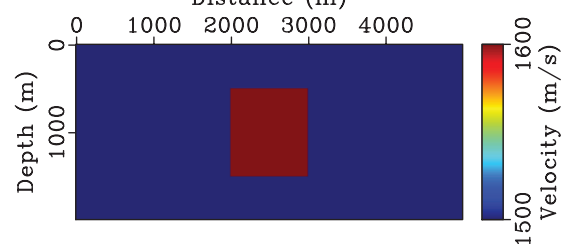

b)

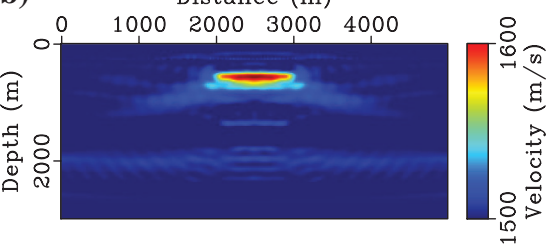

c)

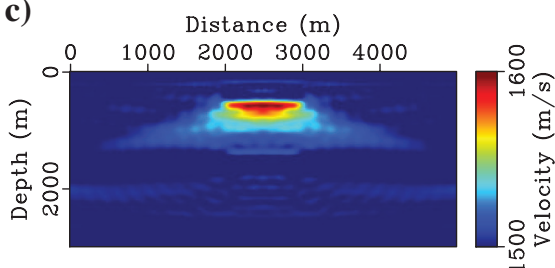

d)

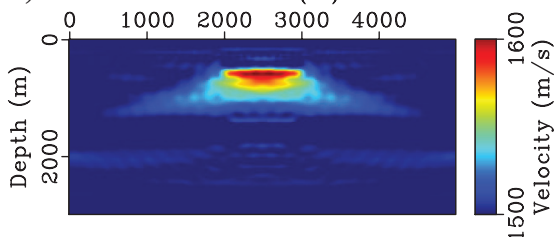

e)

Distance $(\mathrm{m})$

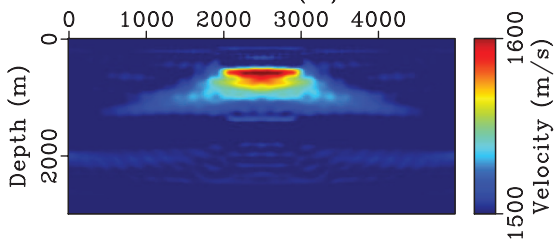

f)

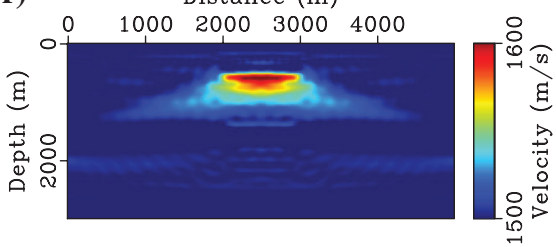

Figure 3. (a) The actual velocity model. (b) The perturbation model for single scattering obtained using the first term of equation 31 . (c) The perturbation model corresponding to using all terms in equation 31 (double scattering). The perturbation model corresponding to (d) triple scattering, (e) fifth-order scattering, and (f) 12th-order scattering. All using 100 iterations of the conjugate-gradient update.

a)

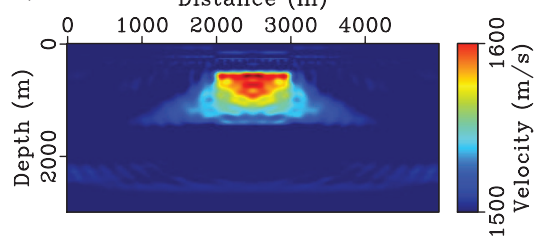

b)

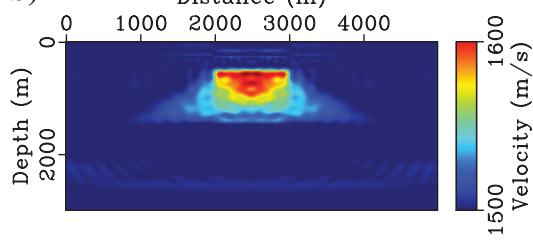

c)

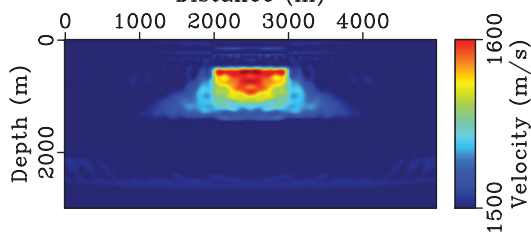

d) Distance $(\mathrm{m})$

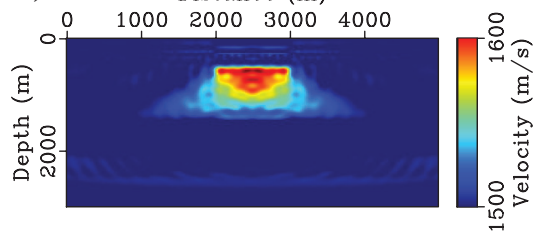

Figure 4. (a) The velocity model after 20 FWI iterations corresponding to $\delta m$ computed using single scattering 100 iterations data fitting. (b) The velocity model after 20 FWI iterations corresponding to $\delta m$ computed using double scattering 100 iterations data fitting. (c) The velocity model after $20 \mathrm{FWI}$ iterations corresponding to $\delta m$ computed using triple scattering 100 iterations data fitting. (d) The velocity model after 20 FWI iterations corresponding to $\delta m$ computed using fifth-order scattering 100 iterations data fitting. 
a spectral time-domain modeling approach that is immune to dispersion problems (Wu and Alkhalifah, 2014) to compute the wavefields. The update of $m_{0}$ will contain only smooth components thanks to the scattering-angle filter, and the initial $\delta m$ is mainly given by the single- and double-scattering image, also cleaned using the scattering-angle filter to remove the high-scattering angles. An optimal result of this RWI/FWI (including MWI) process is given by $m_{0}$ equal to the true velocity and $\delta m=0$. However, $\delta m$ will store the model perturbation components necessary to produce reflections that are not produced by $m_{0}$ due to the inability of the classic FWI to constrain such reflections.

After 100 iterations using an L-BFGS approximation of the Hessian to condition the gradient, we end up with the inverted velocity model shown in Figure 7a. The residual shot gather in Figure 7b shows limited energy. The match of data is provided by the reflections and double scattering produced by the inverted model in Figure $7 \mathrm{a}$ and those corresponding to the final $\delta m$ (image), shown in Figure $7 \mathrm{c}$. This image contains the energy we did not manage to generate using the inverted velocity model. It will usually include the high frequencies and deeper closer to the edge of the model reflections that FWI did not manage to cope with. For comparison, the inverted model using conventional FWI applied to this data set is

a)

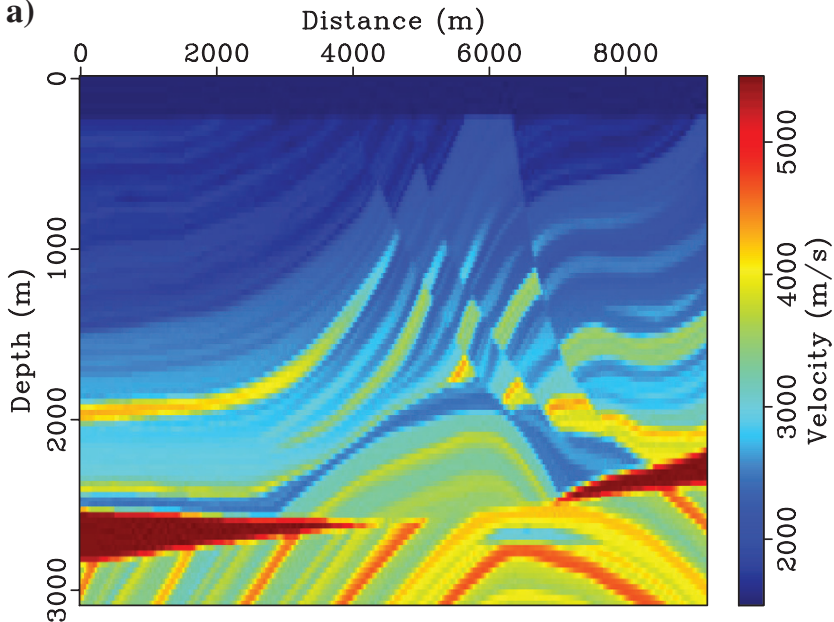

b)

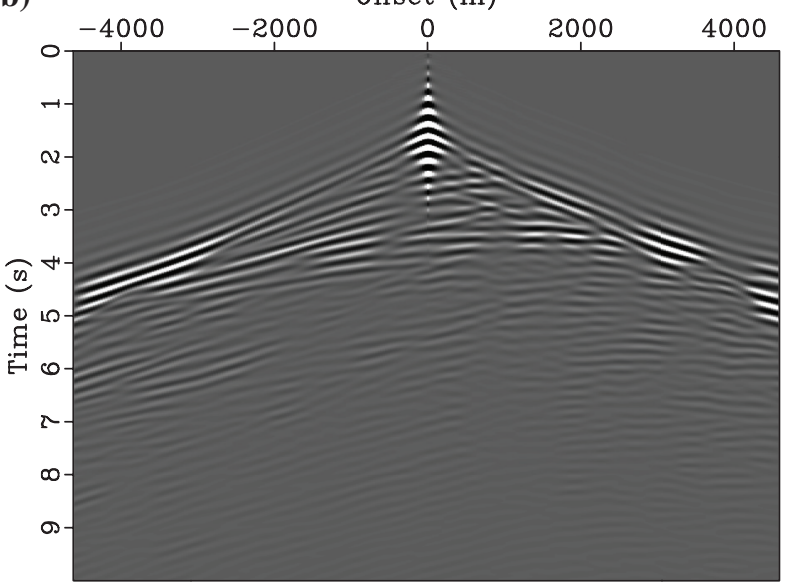

Figure 5. (a) The exact Marmousi model. (b) A shot gather for a shot located at $5000 \mathrm{~m}$. shown in Figure 8a, and it suffers from missing the low frequencies (lower than $5 \mathrm{~Hz}$ ) necessary to avoid falling into a local minima with the initial model. In comparing the single and double scattering, Figure $8 \mathrm{~b}$ shows the inverted model from the previously developed single-scattering (Alkhalifah and Wu, 2016) version of the approach. The model provided by the double scattering shows better velocity definition on the edges of the model due to the additional illumination provided by the double-scattering wavepath updates. The vertical profiles of the models corresponding to locations 3000 (Figure 9a), 5000 (Figure 9b), and $7000 \mathrm{~m}$ (Figure 9c) confirm the accuracy of the inverted model using double scattering (purple curves), when compared with the true model (blue curves). Meanwhile, the conventional FWI admitted poor results especially at depth (green curves).

\section{A SALT CANYON}

Although many of the benefits of addressing multiscattering in our inversion process is realized in the additional wavepath updates, the proper placement of the higher order scattering energy is an additional important feature. We use a piece of the Sigsbee model that includes the salt canyon shown in Figure 10a to demonstrate this

a)

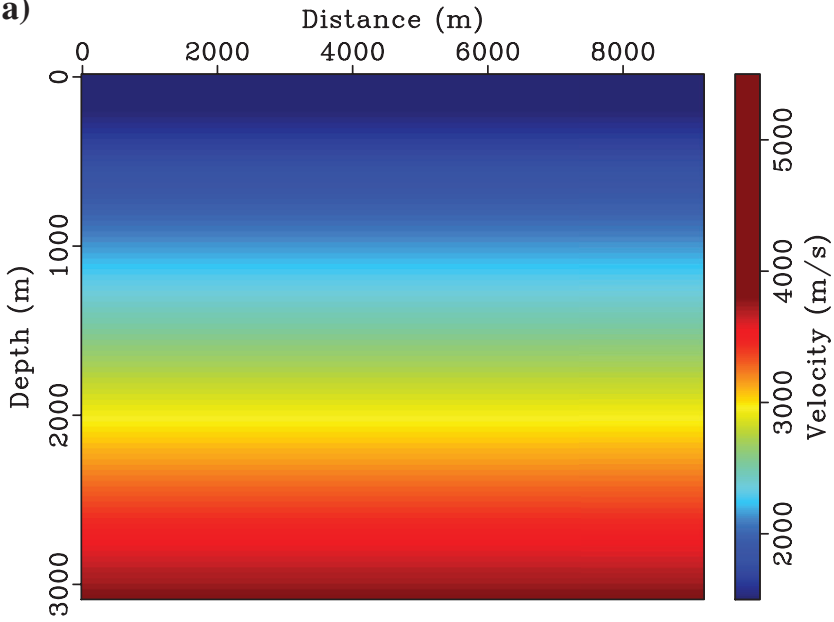

b)

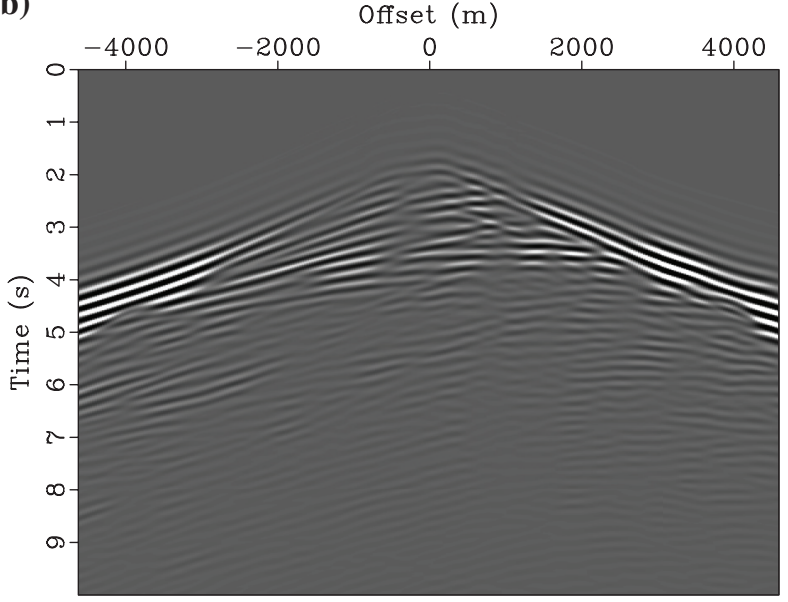

Figure 6. (a) The initial velocity model. (b) The residual shot gather corresponding to the initial model. 
feature. Because the salt is a strong scatterer of seismic energy, the flanks of the canyon induce heavy multiple scattering.

In this experiment, we consider data at a single frequency of $6 \mathrm{~Hz}$. We invert for the $m$ and the $\delta m$ using up to three orders of scattering. The choice of third-order scattering is based on a

a)

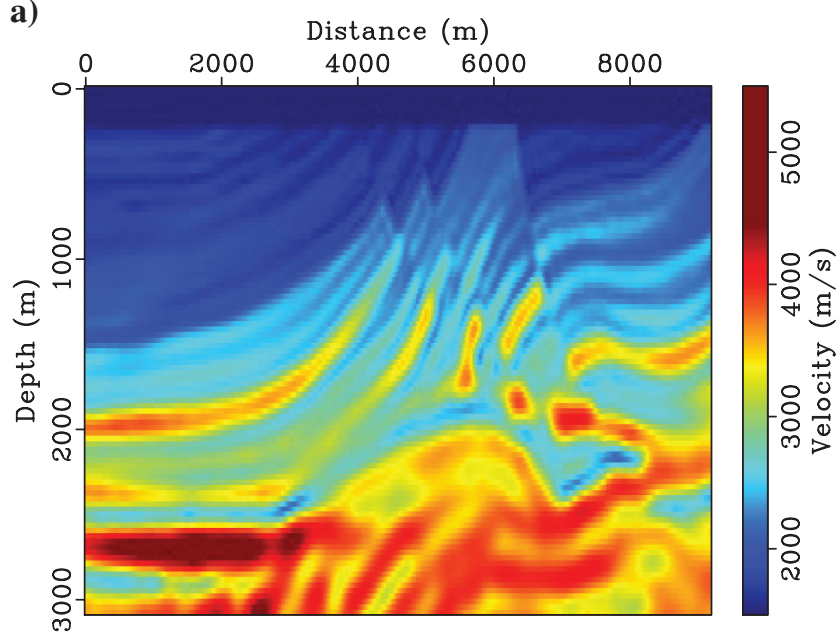

b)

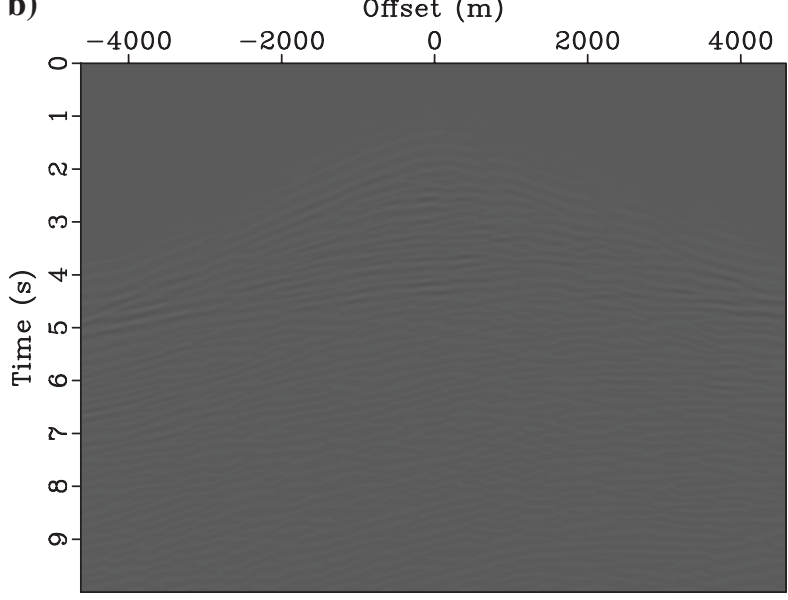

c)

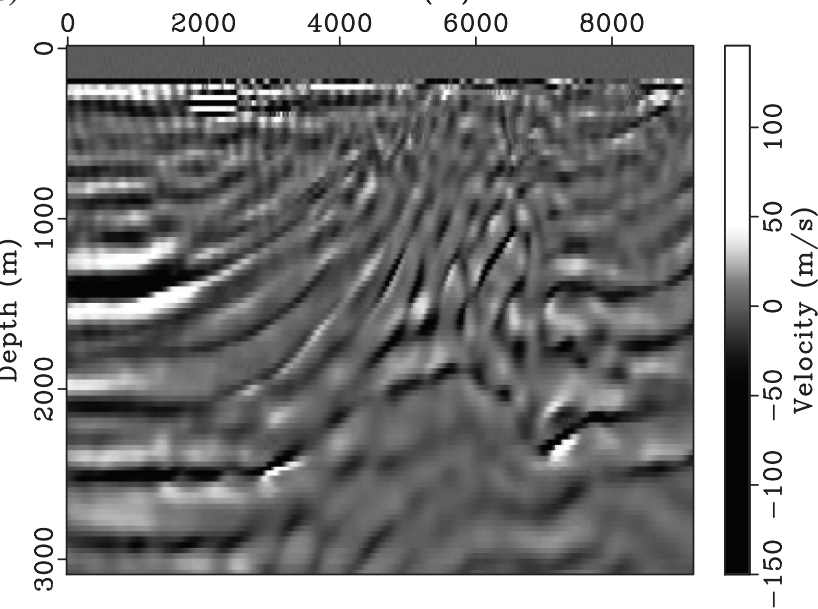

Figure 7. (a) The inverted velocity model using double scattering (objective function 13). (b) The residual shot corresponding to the inverted model. (c) The final image $\delta m$. compromise between accuracy and cost. Every additional scattering order will add mild cost, but this order was reasonable for the saltflank scattering. The model is discretize with a $15 \mathrm{~m}$ spacing laterally and vertically. The shot interval is $60 \mathrm{~m}$, and the receivers are at every gird point on the surface. Our objective here is above the salt and in particular the salt canyon sediments, so we do not update the salt or the water layer. The initial model is given by a linearly increasing velocity under the water bottom (Figure 10b). Because the salt body is not inverted or considered, we add it to the plots of the models throughout to help us identify the inverted regions (above), so that we can focus the evaluation and comparison of the inverted models with the areas above the salt. Figures 11a and $11 \mathrm{~b}$ show the resulting $m$ and $\delta m$, respectively, for this single-frequency inversion $(6 \mathrm{~Hz})$ and third-order scattering-based inversion. The velocity model shows reasonable convergence for this single frequency, as demonstrated in the vertical profiles at locations 1,2 , and $3 \mathrm{~km}$, as shown in Figure $13 \mathrm{a}-13 \mathrm{c}$, respectively. The inverted image also looks reasonably clean using only a single frequency. Meanwhile, for the single-scattering equivalent inversion (RWI), Figure 12a shows worse results, as also demonstrated in the vertical profiles. The corresponding inverted image in Figure $12 b$ is less clean. This result is expected considering the amount
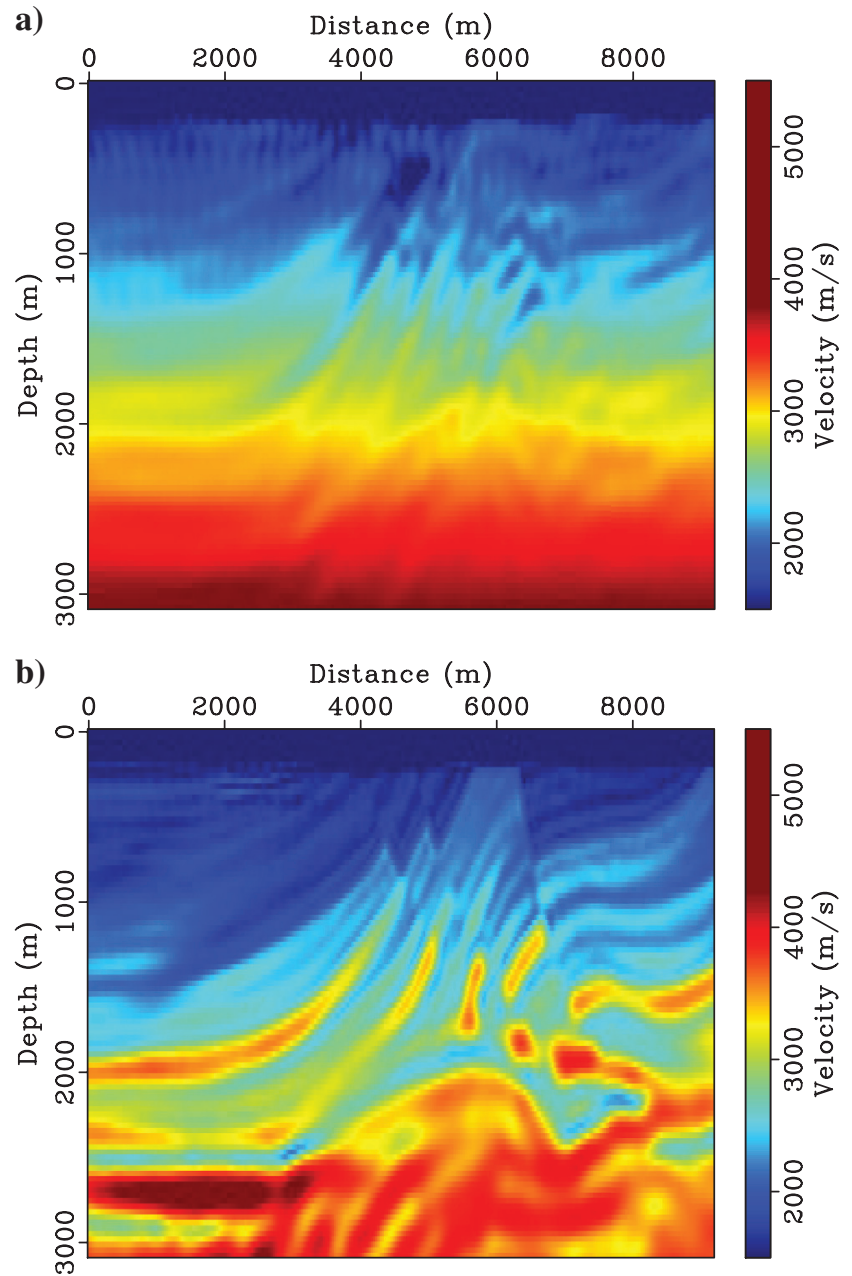

Figure 8. (a) The inverted model from conventional FWI for comparison. (b) The inverted model for a single scattering FWI/RWI. 

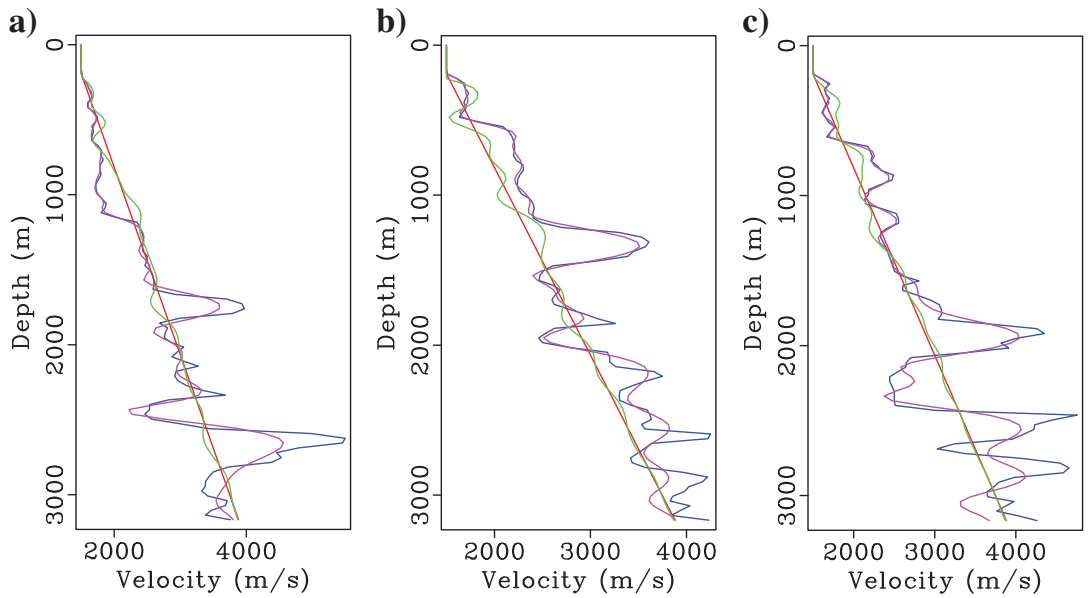

of multiscattering in the canyon between the salt flanks. Thus, the worse part of the singlescattering result is in the canyon region as shown in the profile located at $3 \mathrm{~km}$ (Figure 13c, green).

\section{DISCUSSION}

The inversion for the double scattering $\delta m$ not only provides an approximate full-Hessian update but also properly scales the doublescattering component — a task usually left for the third-order term of the Taylor's series expansion of the objective function. However, in its role to help FWI, and for the box model used here, the difference between using such updates compared with inverting for the single scattering $\delta m$ corresponding to an approximate GaussNewton update is small. The reason is that the update of $m_{0}$ in an iterative fashion ultimately converges to a similar model, and the role of multiscattering is diminished by the $m_{0}$ ability at later iterations to predict it.

Fure 9. Vertical profiles of the true velocity model in Figure 5a (blue), the velocity model in Figure 6a (red), the inverted velocity using double scattering in Fi ure 7a (purple), and inverted velocity model using conventional FWI in Figure 8a (green), for locations (a) 3000, (b) 5000, and (c) $7000 \mathrm{~m}$.

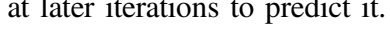

a)

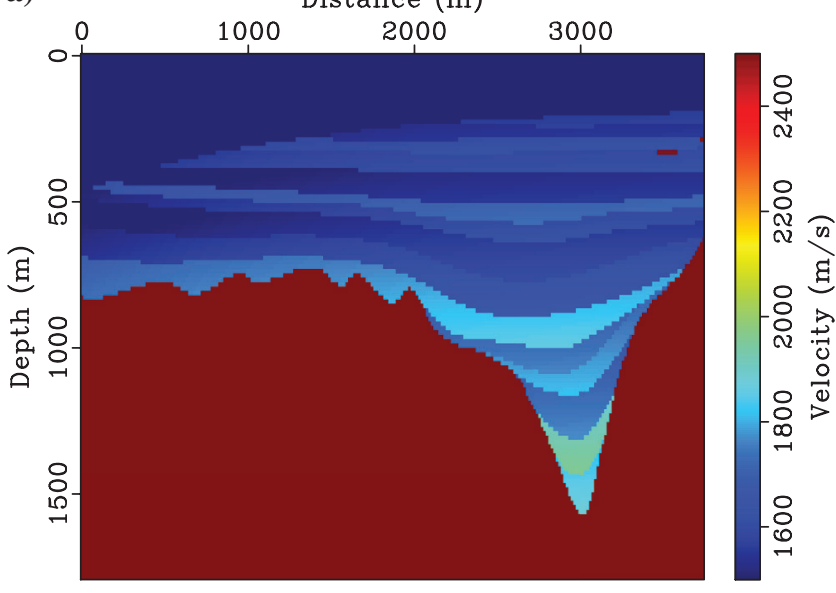

b)

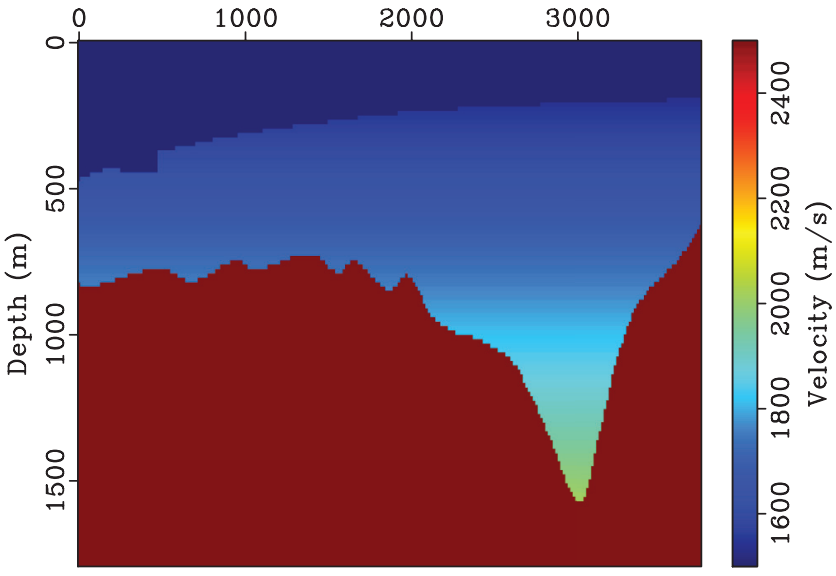

Figure 10. (a) A piece of the Sigsbee model that contains a salt canyon. (b) The initial velocity model. The salt body is added, but not used, in the initial velocity model for better visualization. a)

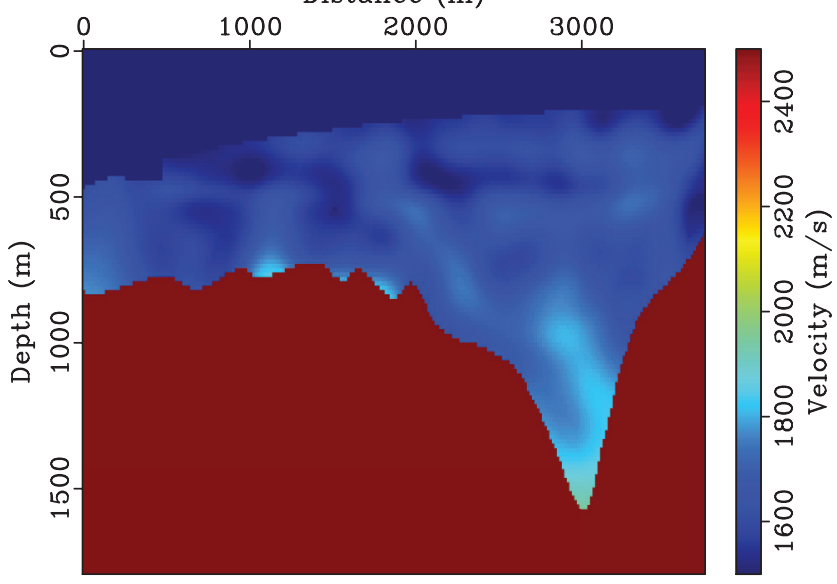

b)

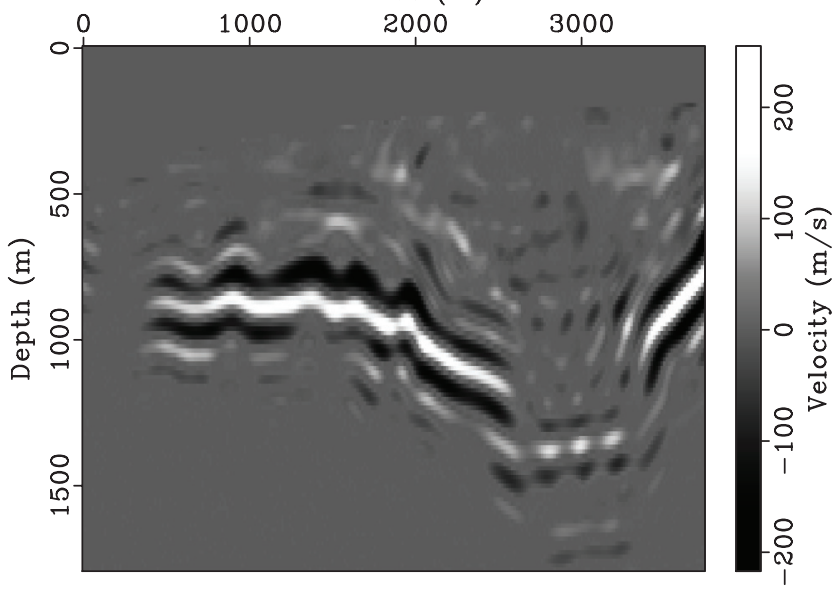

Figure 11. (a) The inverted model $m$ for $6 \mathrm{~Hz}$ frequency data based on the third-order scattering inversion. (b) The inverted perturbation $\delta m$. 

a)

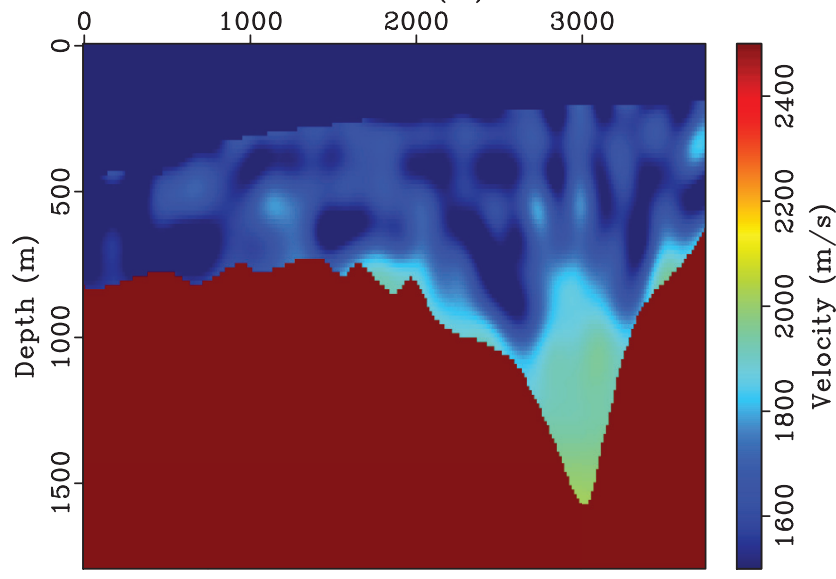

b)

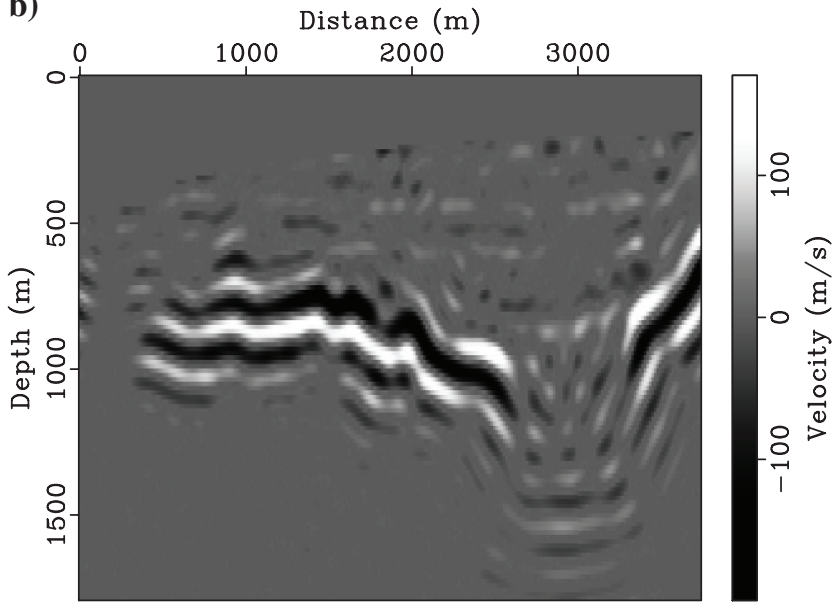

Figure 12. (a) The inverted model $m$ for $6 \mathrm{~Hz}$ frequency data based on the single-scattering inversion. (b) The inverted perturbation $\delta m$.
The real value of multiscattering is provided by the wavepath updates between scatterers. Such wavepath updates include higher resolution information due to the usual short paths between scatterers, as compared with the path back to the source or receiver. Fitting the double-scattering wavepath updates to the data will further improve such updates. Thus, in addition to accurately mapping salt flanks, the additional wavepath information included in multiscattered events provides additional illumination to the model, requiring, in some cases, less-sensor coverage, a feature needed in ocean bottom cable or nodes acquisition.

The inversion for $m_{0}$ and $\delta m$ using the combined FWI/RWI/MWI objective functional and with the scattering-angle filter as a gate keeper provides the opportunity to access low wavenumbers provided by wavepath updates, middle wavenumbers provide by wavepath updates between scatterers, as well as large-offset scattering, and high-wavenumber updates provided by short-offset scattering. The scattering-angle filter initially pushes the high-scattering angles (low wavenumbers) to the $m_{0}$ model and low-scattering angles (high wavenumbers) to the $\delta m$ model. As the background model $m_{0}$ matures, providing accurate propagation to the wavefields, we allow lower scattering angles to contribute to $m_{0}$. The scattering-angle filtering eventually becomes obsolete and $m_{0}$ will hopefully contain all the model components of the true model constrained by the data based on the acoustic isotropic assumption. The components in the data not explained by $m_{0}$ will remain in $\delta m$, including possibly S-wave recorded events.

The multiscattering objective can be applied in the elastic or anisotropic case. In this case, the scattered data based on Born scattering or double scattering can be generated based on perturbations of multiparameters. This opens the door for many options as we deal with vector wavefields and multiparameters. As implementation options increase, the cost of each inversion iteration is expected to increase because we will need additional wavefield extrapolations. However, using a Helmholtz solver, in which most of the cost is upfront in computing the inverse of the stiffness matrix, the cost of computing wavefields corresponding to the same background medium is low. In this case, the difference between conventional FWI and having an optimized single, or even multiscattering does not exceed $50 \%$. After computing the Green's function (the inverse of the stiffness matrix), calculating wavefields is a matrix-vector (source function) multiplication. a)

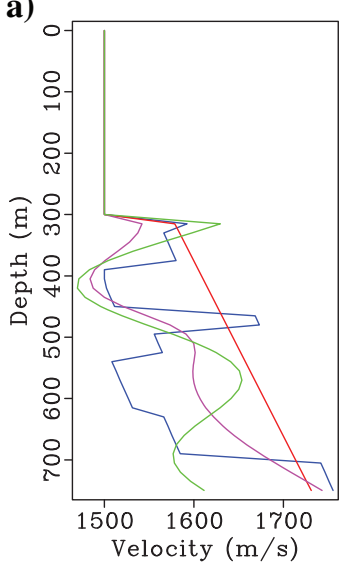

b)

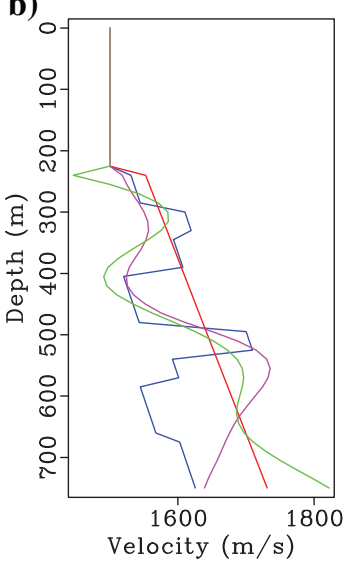

c)

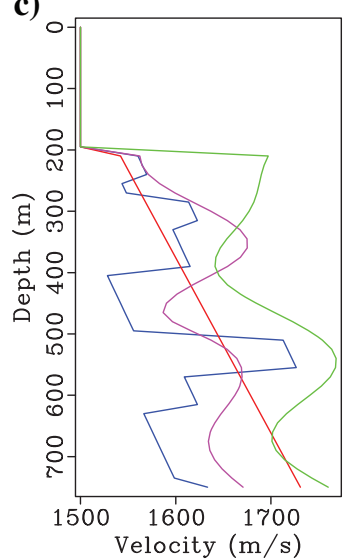

Figure 13. Vertical profiles of the true velocity model in Figure 10a (blue), the initial velocity model (red), the inverted velocity using triple scattering in Figure 11a (purple), and inverted velocity model using single scattering in Figure 12a (green), for locations (a) 1 , (b) 2 , and (c) $3 \mathrm{~km}$.

\section{CONCLUSION}

Using the double (and multiple)-scattering component of the wavefield in inverting for velocity and perturbation models allows us to use more low wavenumbers to build the background model, as well as include the double-scattering energy in fitting the data. In the combined objective functional, the perturbation model provides an image that fits the residual data, and thus, reflection or double-scattering energy not predicted by the velocity model will be generated by the perturbation model. A scattering-angle filter is administrated to insure that we initially admit smooth velocity updates in the initial iterations to the velocity, and clean high-wavenumber components, free of low-frequency artifacts, to the perturbation model. The true value of the inverted double-scattering update is not in its improved 
gradient conditioning, but in its ability to provide additional highresolution wavepath updates between scatterers.

\section{ACKNOWLEDGMENTS}

We thank KAUST for its support. We also thank A. Guitton, J. Cheng, and three anonymous reviewers for their constructive comments and suggestions that ultimately helped to improve the paper.

\section{REFERENCES}

Albertin, U., G. Shan, and J. Washbourne, 2013, Gradient orthogonalization in adjoint scattering-series inversion: 83rd Annual International Meeting, SEG, Expanded Abstracts, 1058-1062.

Alkhalifah, T., 2015a, Conditioning the full waveform inversion gradient to welcome anisotropy: Geophysics, 80, no. 3, R111-R122, doi: 10.1190/ geo2014-0390.1.

Alkhalifah, T., 2015b, Scattering-angle based filtering of the waveform inversion gradients: Geophysical Journal International, 200, 363-373, doi: 10.1093/gji/ggu379.

Alkhalifah, T., and Z. Wu, 2016, The natural combination of full and imagebased waveform inversion: Geophysical Prospecting, 64, 19-30, doi: 10 $.1111 / 1365-2478.12264$

Almomin, A., 2013, Tomographic full waveform inversion (TFWI) by successive linearizations and scale separations: 83rd Annual International Meeting, SEG, Expanded Abstracts, 1048-1052.

Almomin, A., and B. Biondi, 2012, Tomographic full waveform inversion: Practical and computationally feasible approach: 82nd Annual International Meeting, SEG, Expanded Abstracts, doi: 10.1190/segam2012-0976.1.

Berkhout, A. J., 2012, Combining full wavefield migration and full waveform inversion, a glance into the future of seismic imaging: Geophysics, 77, no. 2, S43-S50, doi: 10.1190/geo2011-0148.1.

Bunks, C., F. Saleck, S. Zaleski, and G. Chavent, 1995, Multiscale seismic waveform inversion: Geophysics, 60, 1457-1473, doi: 10.1190/1.1443880.

Chavent, G., and R. Plessix, 1999, An optimal true-amplitude least-squares prestack depth-migration operator: Geophysics, 64, 508-515, doi: 10 .1190/1.1444557.

Claerbout, J. F., 1985, Imaging the earth's interior: Blackwell Scientific Publishers.

Clement, F., G. Chavent, and S. Gómez, 2001, Migration based traveltime waveform inversion of 2D simple structures: A synthetic example: Geophysics, 66, 845-860, doi: 10.1190/1.1444974.

Fleury, C., and F. Perrone, 2012, Bi-objective optimization for the inversion of seismic reflection data: Combined FWI and MVA: 82nd Annual International Meeting, SEG, Expanded Abstracts, doi: 10.1190/ segam2012-0797.1.

Jang, U., D. Min, and C. Shin, 2009, Comparison of scaling methods for waveform inversion: Geophysical Prospecting, 57, 49-59, doi: 10 .1111/j.1365-2478.2008.00739.x.
Khalil, A., J. Sun, Y. Zhang, and G. Poole, 2013, RTM noise attenuation and image enhancement using time-shift gathers: 83rd Annual International Meeting, SEG, Expanded Abstracts, 3789-3793.

Ma, Y., D. Hale, B. Gong, and Z. Meng, 2012, Image-guided sparse-model full waveform inversion: Geophysics, 77, no. 4, R189-R198, doi: 10 .1190/geo2011-0395.1.

Métivier, L., F. Bretaudeau, R. Brossier, S. Operto, and J. Virieux, 2014, Full waveform inversion and the truncated Newton method: Quantitative imaging of complex subsurface structures: Geophysical Prospecting, 62, 1353-1375, doi: 10.1111/1365-2478.12136.

Plessix, R.-E., 2006, A review of the adjoint-state method for computing the gradient of a functional with geophysical applications: Geophysical Journal International, 167, 495-503, doi: 10.1111/j.1365-246X.2006 .02978.x.

Pratt, R., 1999, Seismic waveform inversion in the frequency domain. Part 1: Theory and verification in a physical scale model: Geophysics, 64, 888901, doi: 10.1190/1.1444597.

Pratt, R. G., Z.-M. Song, P. Williamson, and M. Warner, 1996, Two-dimensional velocity models from wide-angle seismic data by wavefield inversion: Geophysical Journal International, 124, 323-340, doi: 10.1111/j .1365-246X.1996.tb07023.x

Sirgue, L., and R. Pratt, 2004, Efficient waveform inversion and imaging: A strategy for selecting temporal frequencies: Geophysics, 69, 231-248, doi: 10.1190/1.1649391.

Symes, W., and M. Kern, 1992, Velocity inversion by differential semblance optimization for 2D common source data: 62nd Annual International Meeting, SEG, Expanded Abstracts, 1210-1213.

Tang, Y., S. Lee, A. Baumstein, and D. Hinkley, 2013, Tomographically enhanced full wavefield inversion: 83rd Annual International Meeting, SEG, Expanded Abstracts, 1037-1041.

Tarantola, A., 1984, Inversion of seismic reflection data in the acoustic approximation: Geophysics, 49, 1259-1266, doi: 10.1190/1.1441754.

Virieux, J., and S. Operto, 2009, An overview of full-waveform inversion in exploration geophysics: Geophysics, 74, no. 6, WCC1-WCC26, doi: 10 $.1190 / 1.3238367$.

Wang, S., F. Chen, H. Zhang, and Y. Shen, 2013, Reflection-based full waveform inversion (RFWI) in the frequency domain: 83rd Annual International Meeting, SEG, Expanded Abstracts, 877-881.

Wu, Z., and T. Alkhalifah, 2014, The optimized expansion based low-rank method for wavefield extrapolation: Geophysics, 79, no. 2, T51-T60, doi: 10.1190/geo2013-0174.1.

Wu, Z., and T. Alkhalifah, 2015, Simultaneous inversion of the background velocity and the perturbation in full-waveform inversion: Geophysics, 80 , no. 6, R317-R329, doi: 10.1190/geo2014-0365.1.

$\mathrm{Xu}, \mathrm{S}$., D. Wang, F. Chen, G. Lambare, and Y. Zhang, 2012, Inversion on reflected seismic wave: 82nd Annual International Meeting, SEG, Expanded Abstracts, doi: 10.1190/segam2012-1473.1.

Zhang, Y., and L. Duan, 2012, Predicting multiples using a reverse time demigration: 82nd Annual International Meeting, SEG, Expanded Abstracts, doi: 10.1190/segam2012-0520.1.

Zhou, W., R. Brossier, S. Operto, and J. Virieux, 2015, Full waveform inversion of diving and reflected waves for velocity model building with impedance inversion based on scale separation: Geophysical Journal International, 202, 1535-1554, doi: 10.1093/gji/ggv228. 